\title{
Association Between a Type 2 Inflammatory Disease Burden Score and Outcomes Among Patients with Asthma
}

\author{
David Price $\mathbb{D}^{1,2}$ \\ Andrew Menzies-Gow ${ }^{3}$ \\ Claus Bachert ${ }^{4}$ \\ Giorgio Walter Canonica ${ }^{5}$ \\ Janwillem Kocks (1D) \\ Asif $\mathrm{H} \mathrm{Khan}^{6}$ \\ Fen $\mathrm{Ye}^{7}$ \\ Paul J Rowe ${ }^{7}$ \\ Yufang Lu ${ }^{8}$ \\ Siddhesh Kamat ${ }^{8}$ \\ Victoria Carter \\ Jaco Voorham ${ }^{1,9}$
}

'Observational and Pragmatic Research Institute (OPRI), Singapore; ${ }^{2}$ Centre of Academic Primary Care, Division of Applied Health Sciences, University of Aberdeen, Aberdeen, Scotland; ${ }^{3}$ Royal Brompton Hospital, London, England; ${ }^{4}$ Upper Airways Research Laboratory, Ghent University, Ghent, Belgium; ${ }^{5}$ Personalized Medicine Asthma and Allergy Center, Humanitas University and Research Hospital, IRCCS, Milan, Italy; ' ${ }^{6}$ Sanofi, Chilly-Mazarin, Paris, France; ${ }^{7}$ Sanofi, Bridgewater, NJ, USA; ${ }^{8}$ Regeneron Pharmaceuticals Inc., Westchester County, NY, USA; ${ }^{9}$ Data to Insights Research Solutions, Lisbon, Portugal
Background: Although prevalence of co-existing type 2 inflammatory diseases (cT2) in asthma patients has been reported, limited data exist regarding their impact on asthma outcomes.

Objective: To assess the impact of cT2 burden on asthma outcomes and to evaluate patterns of clustering of cT2 in a real-world setting.

Methods: From medical records of 4.5 million enrollees in 650 primary care practices in the UK (January 2010-December 2017), patients with $\geq 1$ diagnosis code for asthma at any time pre-index date (date of most recent asthma-related medical encounter) and $\geq 2$ asthma-related prescriptions during the year before index date were categorized into the Global Initiative of Asthma (GINA) guideline severity steps. A cT2 burden score (range 0-9) was assigned based on the total number of co-existing conditions (allergic conjunctivitis, allergic rhinitis, anaphylaxis, eczema/atopic dermatitis, chronic rhinosinusitis, eosinophilic esophagitis, food allergy, nasal polyps, or urticaria) for which patients received a medical diagnosis. Multivariate regression models evaluated associations between cT2 burden score and asthma exacerbations and asthma control. Factor analysis was performed to assess which cT2 comorbidities were correlated and exhibited patterns of clustering.

Results: Overall, 245,893 patients with asthma were included (mean [SD] age 44.8 [22.1] years; $43.8 \%$ male). Between 55\% (GINA step 1) and 60\% (GINA step 5) of asthma patients had a medical diagnosis for $\geq 1$ other type $2 \mathrm{dx}$. Patients with increased cT2 burden were significantly more likely to experience asthma exacerbations and less likely to achieve asthma control.

Conclusion: Asthma patients with a higher cumulative cT2 burden score were more likely to experience worse asthma outcomes than those without any cT2 (burden score of 0).

Keywords: type 2 inflammation, asthma, exacerbations, control, comorbidities, burden

\section{Introduction}

Asthma is a heterogeneous respiratory disorder characterized by chronic airway inflammation with symptoms including wheezing, shortness of breath, chest tightness, and coughing, ${ }^{1,2}$ all of which vary over time and in intensity. Affecting more than 300 million people worldwide, asthma has high epidemiologic, economic, and humanistic burden. ${ }^{2,3}$

Type 2 inflammation, driven by key cytokines including interleukins (IL) $-4,-5$ and -13 - which are produced by T-helper 2 cells and type 2 innate lymphoid cells - is a unifying feature of classically defined allergic and other inflammatory diseases. ${ }^{4,5}$
Correspondence: David Price

Centre of Academic Primary Care,

Division of Applied Health Sciences,

University of Aberdeen, Aberdeen, AB25

2ZD, Scotland

Tel +6568029724

Email dprice@opri.sg 
Approximately $50-70 \%$ of asthma patients have type 2 asthma, which is characterized by type 2 inflammation. ${ }^{6-8}$ The Global Initiative for Asthma (GINA) guidelines 2019 also highlight the type 2 phenotype among patients with severe asthma. ${ }^{9}$ Asthma is increasingly considered to be part of a multimorbidity syndrome, ${ }^{10-12}$ and a large proportion of asthma patients report symptoms of co-existing type 2 inflammatory diseases (cT2) such as eczema/atopic dermatitis, chronic rhinosinusitis with nasal polyposis, eosinophilic esophagitis, allergic rhinitis, or food allergies. ${ }^{13-17}$ Individual cT2 comorbidities can negatively impact patient quality of life and asthma-related outcomes. ${ }^{11,12,18,19}$

Although prevalence of cT2 among asthma patients based on patient self-report has been documented in the literature, limited data exist on characterizing cT2 burden in terms of medical service encounters or medical diagnoses and its impact on asthma outcomes. This real-world study assessed the cT2 disease burden among patients with asthma and evaluated the association between cT2 burden and asthma outcomes, including exacerbations and overall asthma control. Additionally, this study assessed which cT2 comorbidities were correlated and exhibited patterns of clustering of medical service encounters.

\section{Methods}

\section{Study Design}

Anonymized data for the period between January 1, 2010, and December 31, 2017, were extracted from the Optimum Patient Care Research Database (OPCRD), a large UK primary care data source established and maintained by
Optimum Patient Care (OPC), an independent research group. OPCRD contains electronic medical histories for $>4.5$ million enrollees who registered with a primary care provider for receiving outpatient medical services. ${ }^{20}$ Because of the sufficient sample size and a preference for using recent data to better reflect current treatment patterns, data prior to 2010 were not extracted. To enable the comprehensive capture of longitudinal medical history on patients, the date of the last medical encounter for patients at the primary care site or the date of patient disenrollment from the site minus 6 months, whichever came first, was marked as the index date. Figure 1 is a pictographic representation of the index date in relation to the observation window for asthma diagnosis and treatment patterns, medical diagnoses of conditions related to type $2 \mathrm{dx}$ burden, and outcomes of interest.

\section{Inclusion and Exclusion Criteria}

Patients (adults and children $\geq 5$ years) were included in the study if they had $\geq 1$ diagnosis code for asthma at any time before the index date and had $\geq 2$ prescriptions for any asthma medication per GINA guidelines in the year before the index date. All asthma patients were categorized as GINA 1 through 5 severity steps ${ }^{9}$ based on their asthma medication treatment patterns during the year before the index date. If patients stepped-up treatment during the 1-year period, these patients were assigned the highest GINA step.

Patients were excluded if they had any chronic lower respiratory condition other than asthma recorded at any

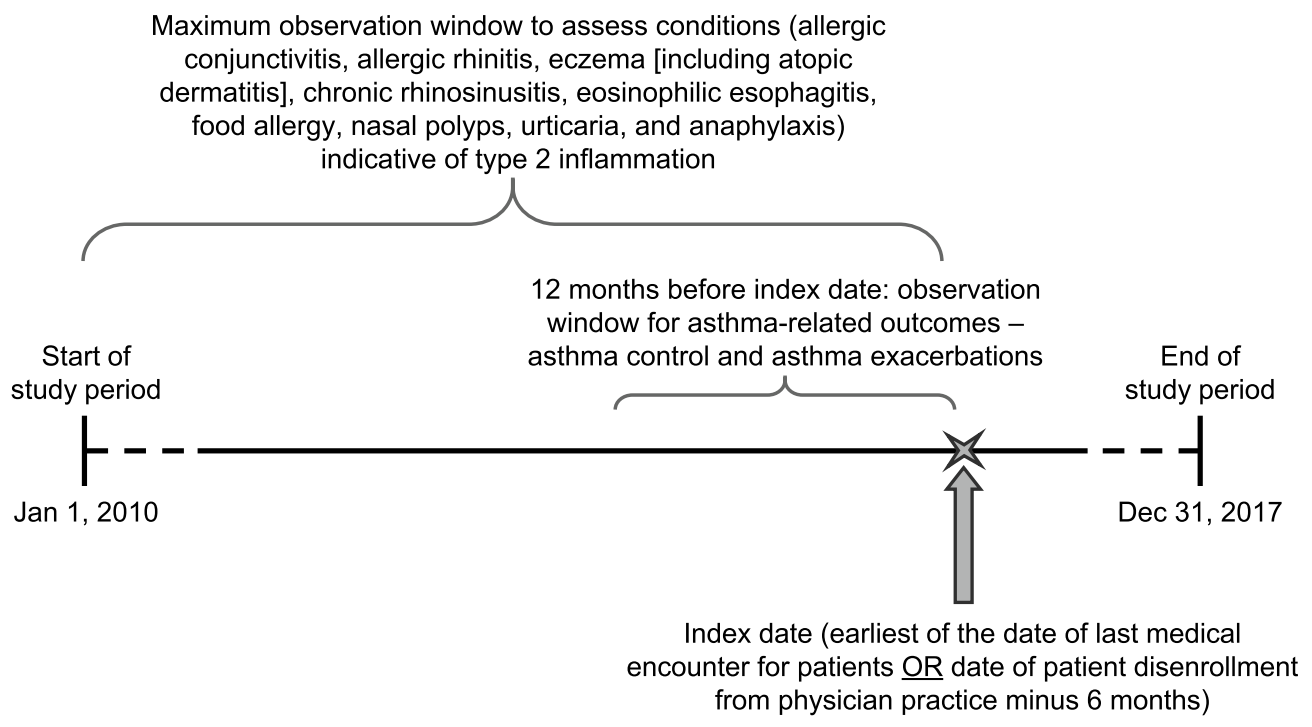

Figure I Retrospective database analysis study design. 
time, including chronic obstructive pulmonary disease, bronchiolitis obliterans, pulmonary fibrosis, pulmonary hypertension, and cystic fibrosis, since these are potential confounding factors. Bronchiectasis was not an exclusion criterion.

\section{Co-Existing Type 2 Inflammatory Disease Burden}

Type $2 \mathrm{dx}$ burden was assessed at any time during the preindex date period based on medical service encounters with Read codes for any one or more of the nine conditions selected a priori based on expert consensus. These conditions included allergic conjunctivitis, allergic rhinitis, anaphylaxis, eczema (including atopic dermatitis), chronic rhinosinusitis, eosinophilic esophagitis, food allergy, nasal polyps, and urticaria. In the OPCRD, the conditions of interest were identified by the presence of Read codes, which are a coded thesaurus of clinical terms that provide standard vocabulary by which clinicians can record patient findings and procedures in health-data systems in the UK. Although some of the conditions listed above may not be cT2 in themselves (eg, anaphylaxis), we assumed that medical service encounters related to these conditions can serve as a measure of cT2 burden. Higher numbers of co-existing cT2 were assumed to reflect higher cT2 burden; for example, patients with Read codes for both chronic rhinosinusitis and nasal polyps were considered to have greater cT2 burden than patients with only one of these medical diagnoses.

\section{cT2 Comorbidity Burden Score and Patterns of Clustering of cT2}

Descriptive statistics of baseline variables were assessed for all asthma patients meeting the study inclusion and exclusion criteria. Continuous variables were summarized using mean, standard deviation, and median. Binary and categorical variables were summarized using the number and percentage of non-missing observations by category.

Each patient was assigned a cT2 burden summary score (ranging from 0 to 9 ) based on the total number of conditions for which patients received medical services; higher scores indicated higher cT2 burden. The association between cT2 burden and asthma-related outcomes was evaluated using multivariable regression models. Finally, factor analysis - an item reduction technique aiming to find the smallest number of factors that can adequately explain the inter-relationships among the variables or items - was performed. This analysis assessed which medical service encounters of the nine comorbidities were correlated with each other and exhibited patterns of clustering.

\section{Outcomes of Interest}

Asthma exacerbations and asthma control were measured for the 12 months before the index date. Severe asthma exacerbations were defined based on the American Thoracic Society (ATS) guidelines, which included the occurrence of one or more of the following: asthmarelated hospital admission or accident and emergency (A\&E) attendance, or an acute course of oral corticosteroids (OCS) for at least 3 days. ${ }^{21}$ Overall asthma control was defined as the absence of any of the following: an asthma-related A\&E attendance, inpatient admission or outpatient department attendance, an acute course of OCS or an antibiotic prescription with a Read code for a lower respiratory consultation; and limited use of shortacting beta- 2 agonists (average daily short-acting $\beta$-agonist dose not exceeding $200 \mu \mathrm{g}$ salbutamol or $>500 \mu \mathrm{g}$ terbutaline). ${ }^{22-25}$

Associations between cT2 burden score (categorized as $1,2,3$, or $4+$ vs 0 as the reference category) and the likelihood of having $\geq 2$ asthma exacerbations and having asthma control was estimated by adjusting for age, gender, and GINA step as covariates in multivariable logistic regression models.

\section{Software}

All data-processing and analyses were performed with Stata SE version 14.2 or Stata MP/6 version 15.1 .

\section{Results}

\section{Baseline Characteristics}

Based on the stepwise selection of patients meeting the inclusion and exclusion criteria, 245,893 asthma patients were included in the study (Figure 2). The mean (standard deviation [SD]) age of patients was 44.8 (22.1); $43.8 \%$ were male and $47.8 \%$ had controlled asthma; most patients (65.1\%) had GINA treatment steps 3-5 (Table 1).

Among the asthma patients in the study cohort, $32.6 \%$ had medical service encounters for allergic rhinitis, $25.8 \%$ for eczema (including atopic dermatitis), 9.0\% for chronic rhinosinusitis, $3.1 \%$ for nasal polyps, and $<0.1 \%$ for eosinophilic esophagitis (Figure 3A includes a list of all nine comorbidities). 


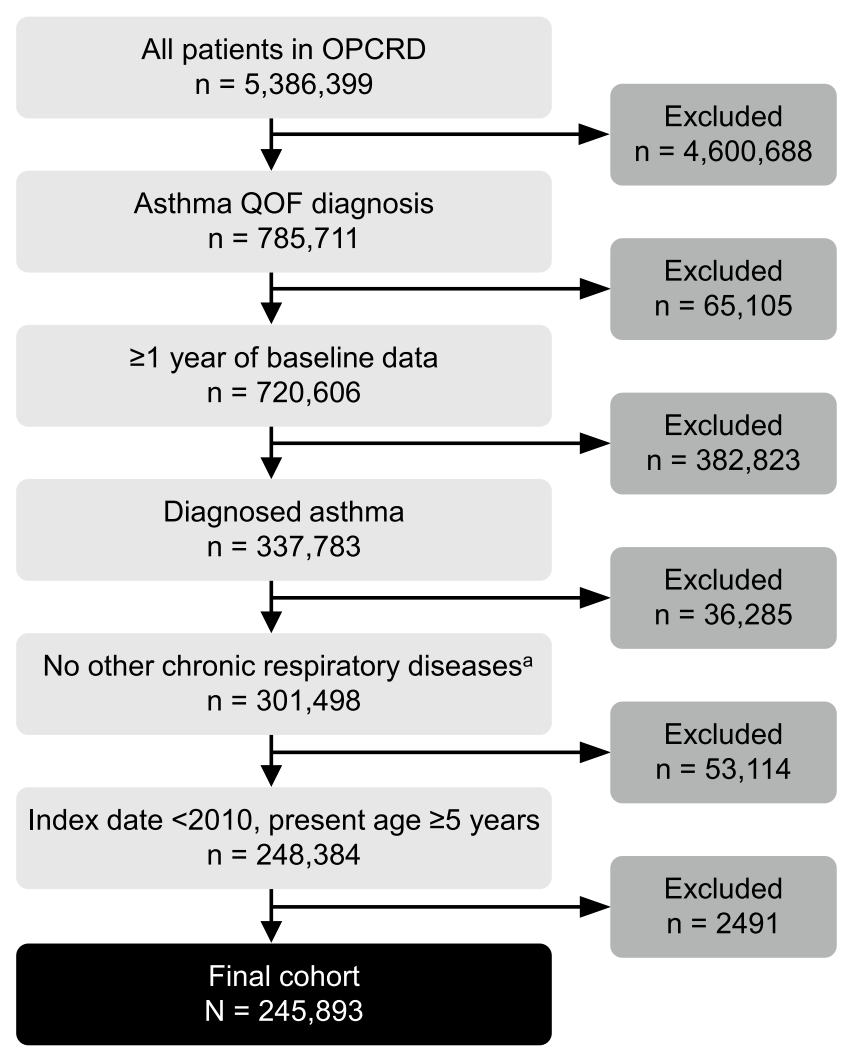

Figure 2 Selection of eligible patients. ' ${ }^{2}$ ung disease due to external agents, other than smoking, such as occupational agents; active chronic obstructive pulmonary disease; bronchiolitis obliterans; pulmonary fibrosis; pulmonary hypertension; cystic fibrosis.

Abbreviations: OPCRD, Optimum Patient Care Research Database; QOF, Quality and Outcomes Framework.

The proportion of patients with a comorbidity burden score of $\geq 1$ was similarly high (56-60\%) among asthma patients across all GINA steps (Figure 3B). However, the proportion of patients with comorbidity burden score of $\geq 2$ and $\geq 3$ increased from GINA step $1(22 \%, 6 \%)$ to GINA step $5(27 \%, 10 \%)$, respectively.

Overall, type2dx burden first peaked in individuals 10 19 years of age and thereafter declined with age. There was a particularly sharp decline in the burden between the age groups 20-29 and 30-39 years, among both males and females (Figure $3 \mathrm{C}$ and $\mathrm{D}$ ).

\section{Asthma Exacerbation Burden and Asthma Control}

As expected, the asthma exacerbation burden increased with increasing GINA steps, ranging from $9 \%$ (step 1) to $29 \%$ (step 5) of patients experiencing $\geq 1$ asthma exacerbation and $2 \%$ (step 1) to $12 \%$ (step 5) of patients experiencing $\geq 2$ asthma exacerbations during the 12 months before the index date (Figure 4A). Similarly, the
Table I Baseline Characteristics

\begin{tabular}{|c|c|}
\hline Variable & $\begin{array}{l}\text { Diagnosed Asthma } \\
\qquad(\mathrm{N}=\mathbf{2 4 5 , 8 9 3 )}\end{array}$ \\
\hline Age (years), mean (SD), median & 44.8 (22.1), 45.0 \\
\hline Male, n (\%) & $107,763(43.8)$ \\
\hline \multicolumn{2}{|l|}{ Smoking status, n (\%) } \\
\hline Never smoked & $140,829(60.5)$ \\
\hline Current smoker & $37,218(16.0)$ \\
\hline Ex-smoker & $54,912(23.6)$ \\
\hline No data on smoking status & $12,934(5.3)$ \\
\hline $\mathrm{FEV}_{1} \%$ predicted, mean $(\mathrm{SD})^{\mathrm{a}}$, median & 85.3 (22.4), 87.0 \\
\hline \multicolumn{2}{|l|}{ GINA treatment step ${ }^{\mathrm{b}}, \mathrm{n}(\%)$} \\
\hline 1 & $23,919(9.7)$ \\
\hline 2 & $61,791(25.1)$ \\
\hline 3 & 61,276 (24.9) \\
\hline 4 & $95,526(38.8)$ \\
\hline 5 & $338 \mathrm{I}(1.4)$ \\
\hline \multicolumn{2}{|l|}{ Exacerbations, ATS definition ${ }^{\mathrm{c}}, \mathrm{n}$ (\%) } \\
\hline 0 & $204,910(83.3)$ \\
\hline $\mathrm{I}$ & $30,420(12.4)$ \\
\hline 2 & $7257(3.0)$ \\
\hline$\geq 3$ & $3306(1.3)$ \\
\hline $\begin{array}{l}\text { Patients having overall asthma control }{ }^{\text {, }} \text {, } \\
\text { n (\%) }\end{array}$ & II 7,423 (47.8) \\
\hline
\end{tabular}

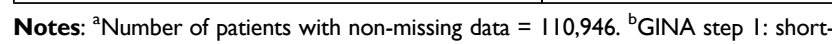
acting relievers only; GINA step 2: low-dose inhaled corticosteroid (ICS) without controllers, leukotriene receptor (LTRA) without controllers, theophylline without controllers, sodium cromoglicate, or nedocromil sodium; GINA step 3: medium- or high-dose ICS without controllers, low-dose ICS/long-acting beta agonist (LABA), lowdose ICS/long-acting muscarinic antagonist (LAMA), low-dose ICS without LABA or LAMA plus LTRA and/or theophylline, LABA and/or LAMA without ICS, LTRA plus theophylline without ICS; GINA step 4: medium- or high-dose ICS/LABA, medium- or high-dose ICS/LAMA, medium- or high-dose ICS plus LTRA and/or theophylline, lowdose ICS/LABA plus $\geq 1$ controller, low-dose ICS/LAMA plus $\geq 1$ controller, $\geq 3$ controllers without ICS; GINA step 5: maintenance OCS plus other treatment or antiimmunoglobulin-E therapy. Includes one or more occurrences of any of the following: asthma-related hospital admission or A\&E attendance or an acute course of oral corticosteroids. ${ }^{\mathrm{d}}$ Controlled asthma, defined as the absence of the following: an asthma-related A\&E attendance, inpatient admission or outpatient department attendance, an acute course of oral corticosteroids with evidence of a lower respiratory consultation, an antibiotic prescribed with evidence of a lower respiratory consultation; and limited use of short-acting beta- 2 agonists (average daily short-acting $\beta$-agonist dose not exceeding $200 \mu \mathrm{g}$ salbutamol or $>500 \mu \mathrm{g}$ terbutaline).

Abbreviations: ATS, American Thoracic Society; $\mathrm{FEV}_{1}$, forced expiratory flow in I second; GINA, Global Initiative for Asthma; SD, standard deviation.

proportion of patients with uncontrolled asthma increased from 53\% (step 1) to $75 \%$ (step 5) (Figure 4B).

\section{Association Between Type 2 Inflammatory Disease Burden and Asthma Outcomes}

Based on results of multivariable regression analysis (after controlling for covariates including patient age, gender, and 
A

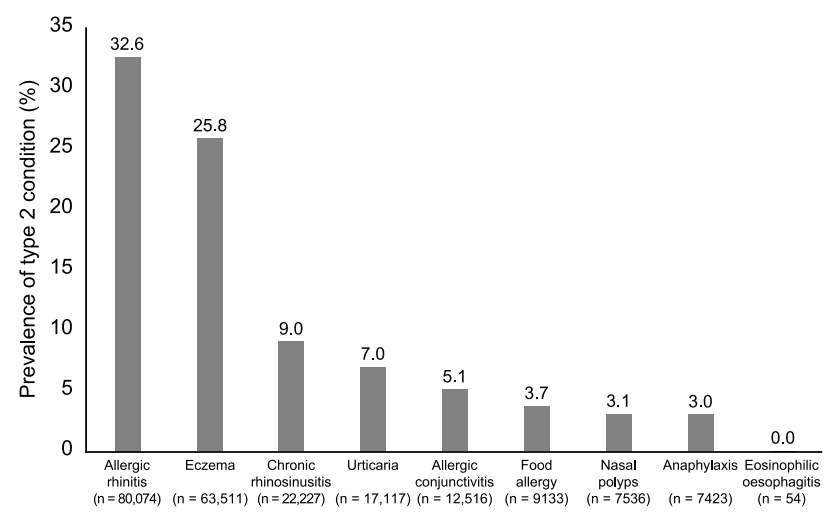

B

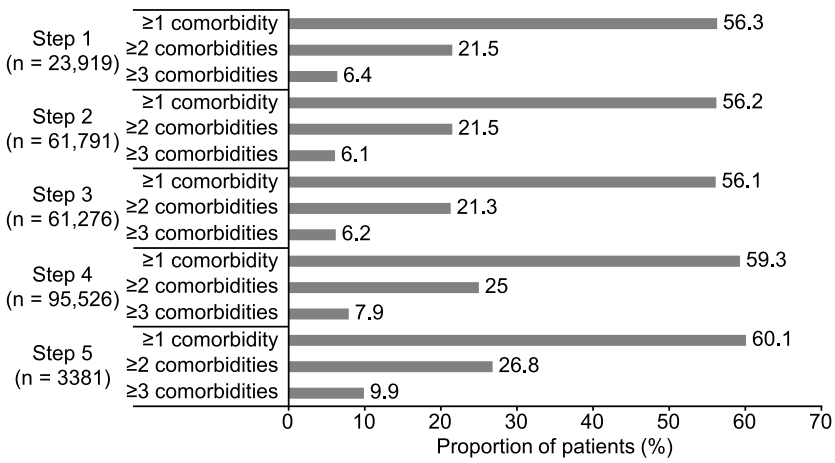

Comorbidities were not mutually exclusive, and patients could contribute to more than one of the above categories.
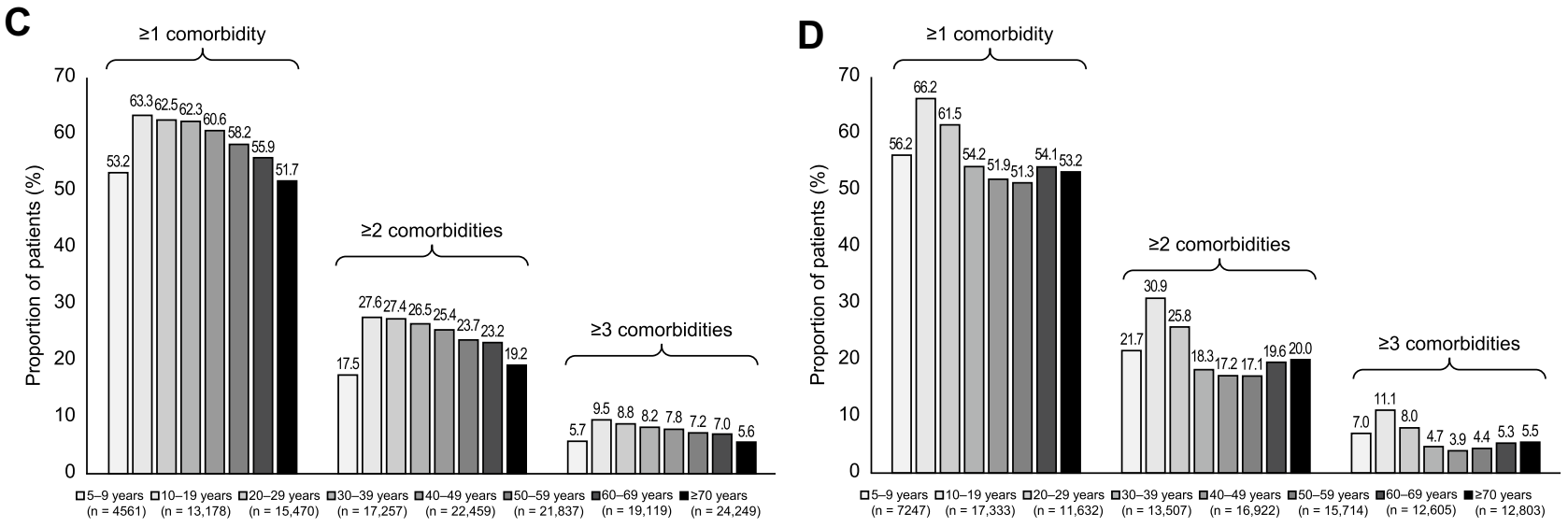

Figure 3 (A) Proportion of asthma patients with medical encounters related to type 2 comorbidities. (B) Comorbidity burden score by GINA steps among asthma patients. (C) Comorbidity burden score by age category in females. (D) Comorbidity burden score by age category in males.

Abbreviation: GINA, Global Initiative for Asthma.

GINA step), patients with a higher cT2 burden score were significantly more likely to experience $\geq 2$ asthma exacerbations (2 comorbidities: odds ratio [OR] 1.16 [1.10-1.23]; 3 comorbidities: OR 1.35 [1.24-1.47]; $\geq 4$ comorbidities: OR 1.74 [1.52-1.95]; Figure 5A) and significantly less likely to achieve asthma control ( 2 comorbidities: OR 0.86 [0.830.88]; 3 comorbidities: OR 0.77 [0.74-0.88]; $\geq 4$ comorbidities: OR 0.67 [0.63-0.72]; Figure 5B) compared with patients without any type 2 inflammatory diseases (ie, cT2 burden score of 0 , reference group).

\section{Clustering of Medical Encounters for the Nine a Priori Conditions Related to Type2dx Burden}

Bivariate analysis was conducted to estimate the OR for the occurrence of medical diagnosis for each of the nine conditions with every other condition. Asthma patients with a diagnosis of nasal polyps were also more likely to have a diagnosis of allergic rhinitis and chronic rhinosinusitis. Asthma patients with codes for food allergies were also more likely to have codes for anaphylaxis and eczema (see Figure S1A). Additionally, asthma patients with codes for urticaria were also more likely to have codes for eczema, food allergy, anaphylaxis, and allergic rhinitis (Figure S1A-H).

When all nine conditions were evaluated in a singlefactor analysis to assess clustering of any medical service encounters related to cT2, it was revealed that patients with asthma tended to have type $2 \mathrm{dx}$ disease burden in three distinct clusters: the first cluster, comprising food allergy and anaphylaxis; the second cluster, comprising chronic rhinosinusitis and nasal polyps; and the third cluster, which included allergic rhinitis, eczema, allergic conjunctivitis, and urticaria (Figure 6).

\section{Discussion}

Type 2 asthma is a widely prevalent type of persistent, uncontrolled asthma ${ }^{26}$ and includes allergic asthma and 

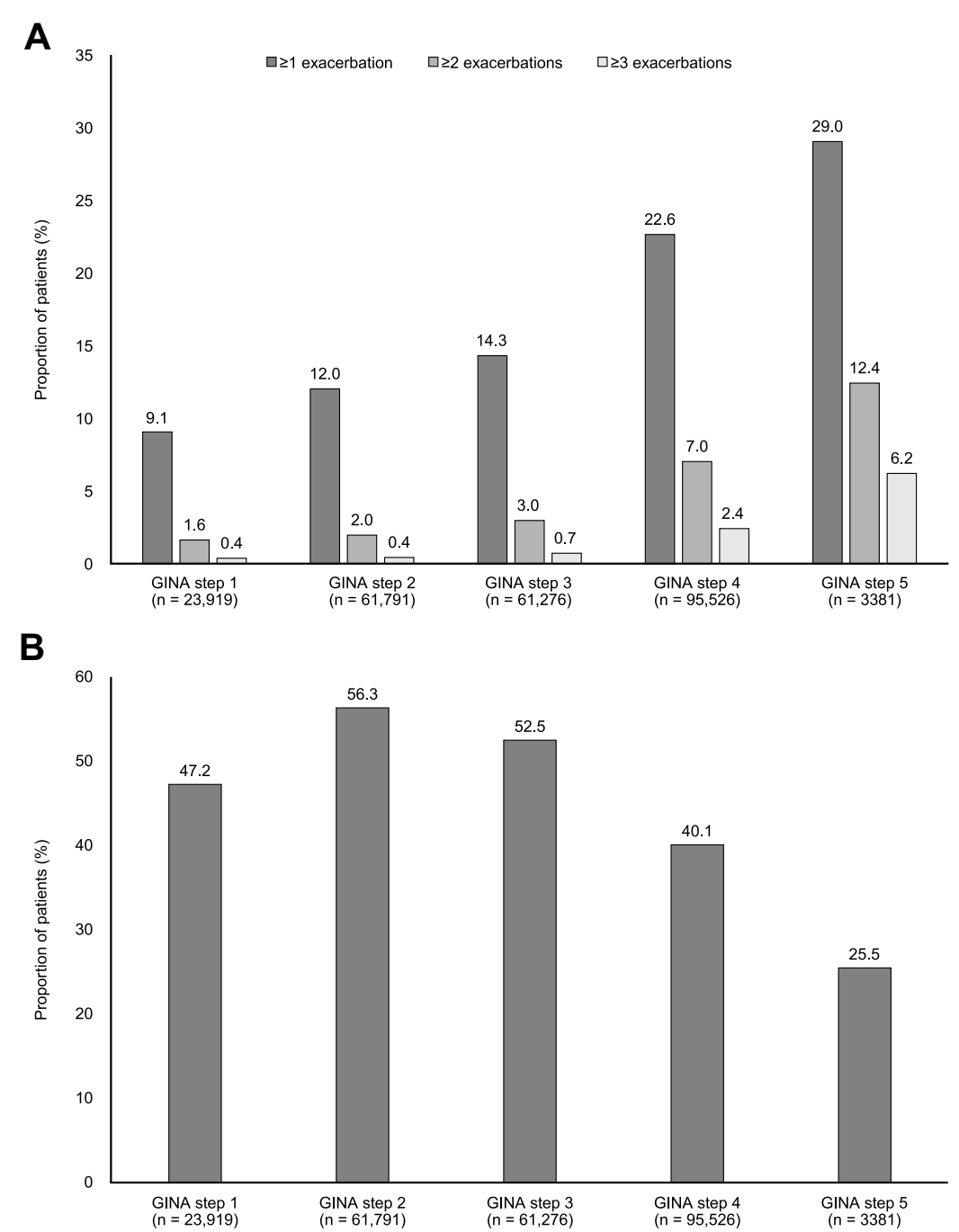

Figure 4 Outcomes of interest categorized by GINA step. (A) Proportion of patients with asthma exacerbation. (B) Proportion of patients having overall asthma control. Abbreviation: GINA, Global Initiative for Asthma.

eosinophilic asthma phenotypes, which have overlapping pathophysiological mechanisms. ${ }^{8}$ It is well established that IL-4 and IL-13 are key drivers of cT2 such as atopic dermatitis, allergic rhinitis, nasal polyps, and eosinophilic esophagitis. ${ }^{27,28}$ Although prevalence of cT2 among patients with asthma has been reported based on patient self-reports, this study provides insights into the cT2 burden in terms of medical diagnosis of conditions and service encounters related to cT2 among patients with asthma.

While the impact of cT2 such as allergic rhinitis on healthcare resource utilization among asthma patients has been previously investigated, ${ }^{29-31}$ few studies, to our knowledge, have investigated the impact of overall cT2 burden on asthma exacerbations and asthma control. One cross-sectional, observational study found that co-existing allergic rhinitis was associated with an incremental adverse impact on the level of asthma control; ${ }^{32}$ meanwhile, a second cross-sectional, observational study found that patients reporting severe rhinitis had worse asthma control than those with mild or no rhinitis symptoms. ${ }^{33}$ The trends observed in these two cross-sectional studies are reflected in our study, ${ }^{32}$ as asthma patients with an increased number of cT2 had decreased asthma control compared with asthma patients with fewer cT2. However, we believe that our study is the first to reveal the impact of multiple cT2 on asthma control, beyond allergic rhinitis.

Results of this study also confirm that asthma patients with cT2 had a higher risk of experiencing severe exacerbations compared with asthma patients with fewer cT2, which is supported by previously published evidence 


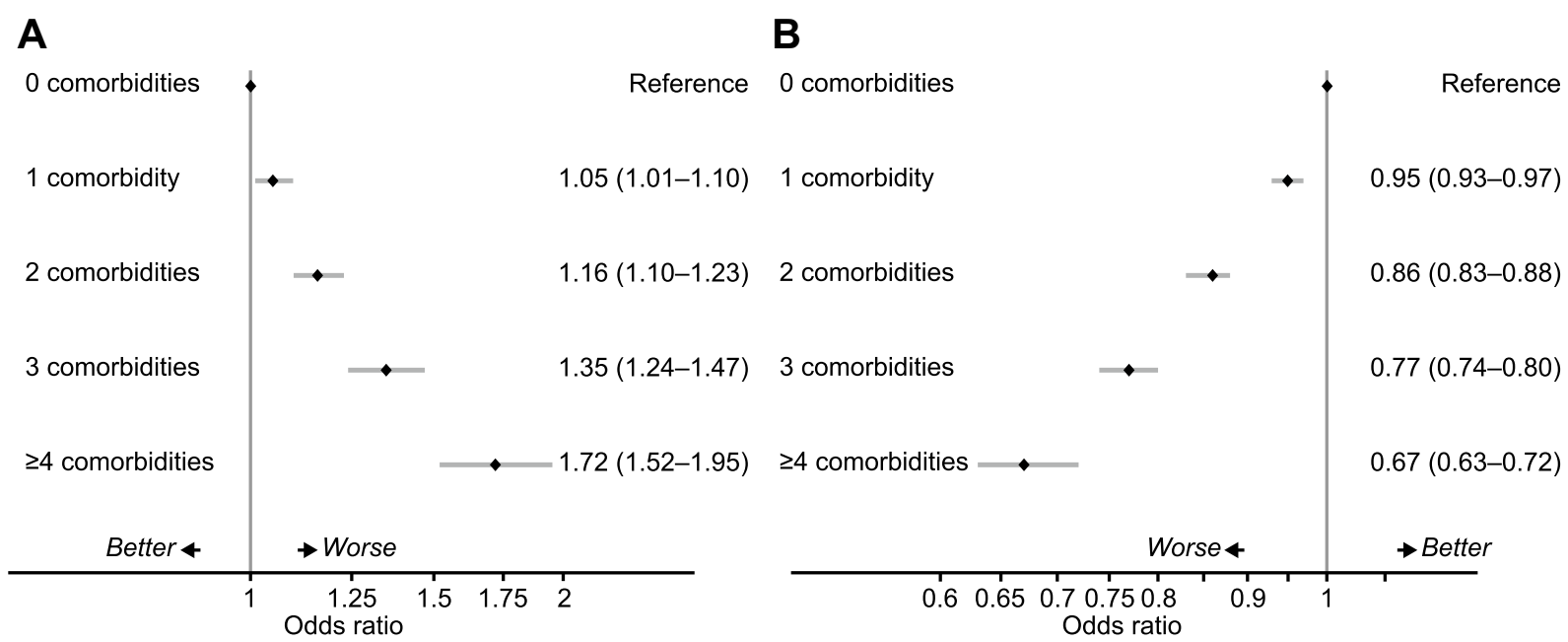

Figure 5 (A) Odds ratios for relationship between type 2 disease-related comorbidity burden score and asthma exacerbations. (B) Odds ratios for type 2 disease-related comorbidity burden score and achievement of asthma control. Logistic regression analysis was conducted using the following covariates: patient age, gender, and Global Initiative for Asthma (GINA) step.

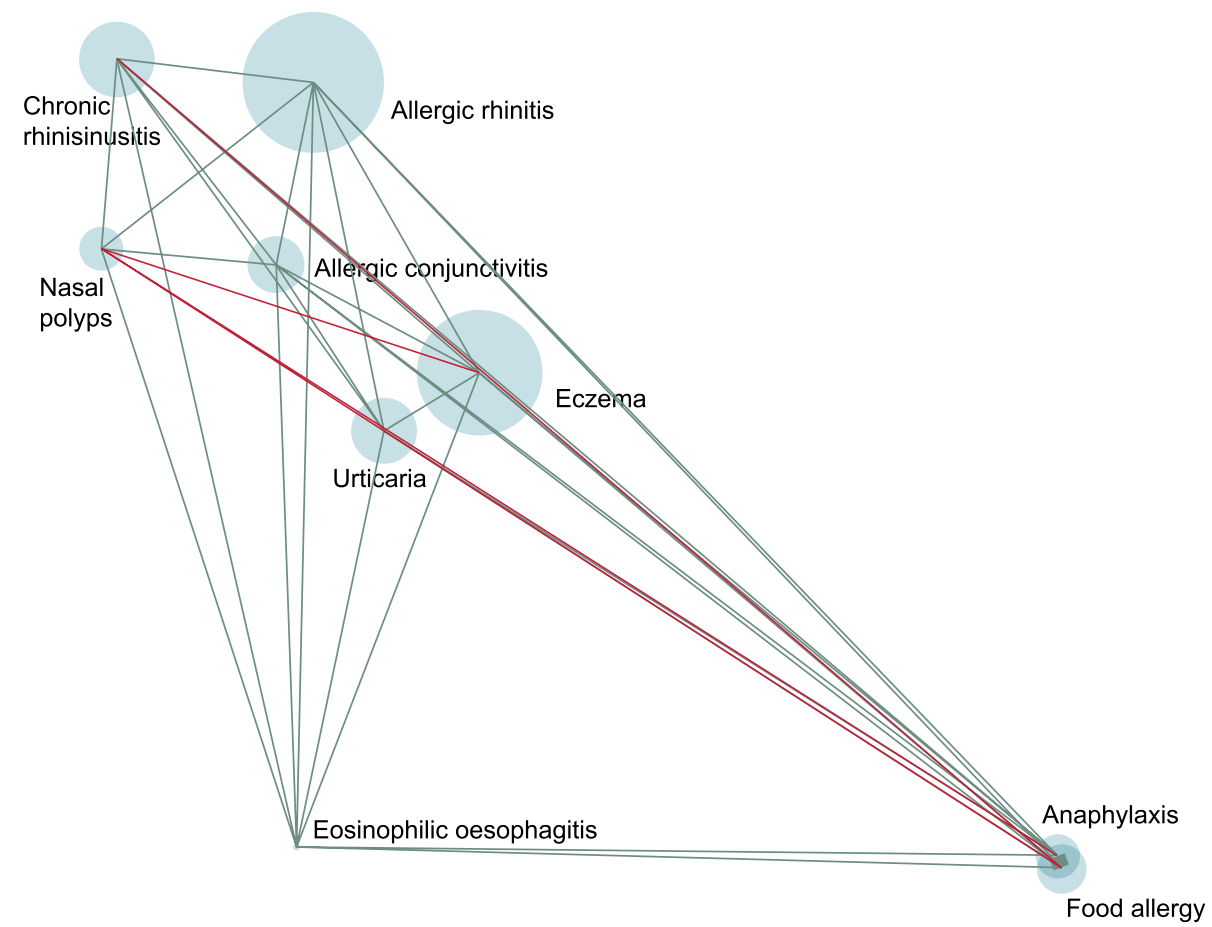

Figure 6 Clustering of medical service encounters related to type 2 inflammatory diseases among patients with asthma. Size of circles is proportional to the prevalence of the comorbidity. The width of the lines connecting the two comorbidities is proportional to the strength of their association. The color of the lines indicates the direction of association: positive (teal) or negative (red). The position of the comorbidities is based on the rotated eigenvalues from the Principal Component Analyses, and shows the nearness based on co-occurrence frequencies and association strengths and directions.

demonstrating the association between asthma and comorbid allergic rhinitis and increased exacerbations. ${ }^{34,35}$ Among asthma patients, the burden of cT2 first peaked between the age 5-9 and age 10-19 cohorts, then appeared to decline across the older age groups. This trend is not unusual as it is understood that some children can outgrow
cT2 such as eczema ${ }^{36}$ and food allergies. ${ }^{37}$ Burden of type 2 comorbidities was high across all GINA steps, with the majority of asthma patients having a diagnosis for at least one other cT2.

Previous studies have demonstrated that allergic rhinitis is a prevalent comorbidity among patients with 
asthma, ${ }^{38-43}$ and even more so among patients with uncontrolled moderate-to-severe asthma, with $<74 \%$ of these patients also presenting with allergic rhinitis. ${ }^{44}$ In this study, associations between comorbidities, based on the likelihood ratios of occurrence, were very strong for anaphylaxis and food allergy, and strong between chronic rhinosinusitis and nasal polyps. The strong association between anaphylaxis and food allergy observed in this study is not surprising, as food allergy is a common cause of anaphylaxis. ${ }^{45}$ Additionally, similar to our findings in primary care, the strong association between medical diagnosis of chronic rhinosinusitis and nasal polyps is well documented for other settings, since nasal polyps is a phenotype of chronic rhinosinusitis (clinical trials, health surveys, and multicenter observational studies). ${ }^{13-15,46}$

A key strength of the current study is the use of realworld, population-based data and the large sample size. ${ }^{47}$ However, our study findings must also be interpreted in light of some limitations. As is the case with real-world studies, which use healthcare service encounter databases, this analysis will under-represent loss of asthma control during situations in which the patient already had a prescription of an OCS or SABA on-hand, which was subsequently used during an episode of worsening in asthma symptoms. Because this study used service encounter data, to the extent that physicians use READ codes for eczema/AD and urticaria/atopy interchangeably, it is not possible to differentiate between these conditions. It was not possible to determine how the clustering of type comorbidities differed according to early- and late-onset asthma. The OPCRD did not include information on the severity of the cT2; hence, the novel comorbidity burden score developed in this study only captured disease burden in terms of the number of cT2 rather than both the number and severity of conditions related to type 2 inflammation. Additionally, it is possible that there was an under-representation of the disease burden due to the lack of a standardized usage of Read codes for specific cT2 and asthma, or because patients may have been self-managing some of the conditions, especially mild rhinitis and eczema, with over-thecounter medications. The resulting data from the prespecified analyses in this study suggest that the diagnosis of urticaria as used by clinicians increases the risk of asthma exacerbations and, to some extent, validates it. Additionally, results from a previous study suggest that at least chronic spontaneous urticaria is associated with increased $\mathrm{T} 2$ markers. $^{48}$ Although urticaria may be accompanied by angioedema or angioedema may occur independently, angioedema was not included in the a priori analysis plan. Therefore, it is possible that inclusion of both components separately may provide additional information related to cT2. Another limitation is that food allergy may be overestimated in the current study; however, it is associated with increased asthma exacerbation risk.

\section{Conclusions}

This real-world, retrospective database study has demonstrated that patients with asthma, irrespective of GINA step, received medical service encounters related to cT2, thereby increasing resource use among asthma patients. The presence of type 2 comorbidities is associated with a higher risk of exacerbations and lower asthma control. Given that the presence of cT2 complicates the management of asthma and is associated with higher risk of exacerbations and lower asthma control, clinicians may consider the assessment of type 2 comorbidity burden in their evaluation of patients with moderate-to-severe asthma.

\section{Abbreviations}

A\&E, accident and emergency; ATS, American Thoracic Society; cT2, co-existing type 2 inflammatory diseases; $\mathrm{FEV}_{1}$, forced expiratory flow in 1 second; GINA, Global Initiative for Asthma; ICS, inhaled corticosteroid; IL, interleukin; LABA, long-acting beta agonist; LAMA, long-acting muscarinic antagonist; LTRA, leukotriene receptor; OCS, oral corticosteroid; OPCRD, Optimum Patient Care Research Database; OR, odds ratio; QOF, Quality and Outcomes Framework; SD, standard deviation.

\section{Ethics Statement}

The OPCRD is approved by the UK National Health Service for clinical research use (Research Ethics Committee reference: 15/EM/0150). The current study protocol was approved by the Anonymized Data Ethics \& Protocol Transparency committee (ADEPT0318), performed in compliance with Good Clinical Practice and Good Pharmacoepidemiology Practice and registered with the European Union electronic Register of PostAuthorization studies (EUPAS25203). 


\section{Acknowledgments}

Medical writing support was provided by Abby Armitt, MSc, and Gauri Saal, MA Economics, of Prime, Knutsford, UK, and funded by Sanofi and Regeneron Pharmaceuticals, Inc.

\section{Author Contributions}

All authors made a significant contribution to the work reported, whether that is in the conception, study design, execution, acquisition of data, analysis and interpretation, or in all these areas; took part in drafting, revising or critically reviewing the article; gave final approval of the version to be published; have agreed on the journal to which the article has been submitted; and agree to be accountable for all aspects of the work.

\section{Funding}

This study was sponsored by Sanofi and Regeneron Pharmaceuticals, Inc.

\section{Disclosure}

David Price: Board membership with AstraZeneca, Boehringer Ingelheim, Chiesi, Mylan, Novartis, Regeneron Pharmaceuticals, Sanofi Genzyme, Thermofisher; consultancy agreements with Airway Vista Secretariat, AstraZeneca, Boehringer Ingelheim, Chiesi, EPG Communication Holdings Ltd, FIECON Ltd, Fieldwork International, GlaxoSmithKline, Mylan, Mundipharma, Novartis, OM Pharma SA, PeerVoice, Phadia AB, Spirosure Inc, Strategic North Limited, Synapse Research Management Partners S.L., Talos Health Solutions, Theravance and WebMD Global LLC; grants and unrestricted funding for investigator-initiated studies (conducted through Observational and Pragmatic Research Institute Pte Ltd) from AstraZeneca, Boehringer Ingelheim, Chiesi, Mylan, Novartis, Regeneron Pharmaceuticals, Respiratory Effectiveness Group, Sanofi Genzyme, Theravance and UK National Health Service; payment for lectures/speaking engagements from AstraZeneca, Boehringer Ingelheim, Chiesi, Cipla, GlaxoSmithKline, Kyorin, Mylan, Mundipharma, Novartis, Regeneron Pharmaceuticals and Sanofi Genzyme; payment for travel/accommodation/meeting expenses from AstraZeneca, Boehringer Ingelheim, Mundipharma, Mylan, Novartis, Thermofisher; stock/ stock options from AKL Research and Development Ltd which produces phytopharmaceuticals; owns $74 \%$ of the social enterprise Optimum Patient Care Ltd (Australia and UK) and 92.61\% of Observational and Pragmatic Research Institute Pte Ltd (Singapore); 5\% shareholding in Timestamp which develops adherence monitoring technology; is peer reviewer for grant committees of the UK Efficacy and Mechanism Evaluation programme, and Health Technology Assessment; and was an expert witness for GlaxoSmithKline. Andrew Menzies-Gow: attended advisory boards for AstraZeneca, Sanofi, Teva, Novartis, Boehringer Ingelheim, and GlaxoSmithKline. He has received lecture fees from Novartis, Teva, Vectura, Boehringer Ingelheim, and AstraZeneca. He has attended international conferences with Teva. He has consultancy agreements with Sanofi, AstraZeneca, and Vectura. Claus Bachert: supported by grants from the European Commission's Seventh Framework programme under grant agreement no. 260895 (PREDICTA); from the Flemish Scientific Research Board, FWO, No. A12/ 5-HB- KH3, by the Global Allergy and Asthma European Network (GA2LEN) project G.0436.04, 3G.0489, G.0642.10N; and by the Interuniversity Attraction Poles Program-Belgian State Belgian Science Policy, No. IAP P7/30. He also served as speaker for Sanofi, Novartis, GlaxoSmithKline, AstraZeneca and Mylan. Giorgio Walter Canonica: received grants and unrestricted funding for investigator-initiated studies and independent research from AstraZeneca, Chiesi, GSK, ALK, Allergy Therapeutics, Novartis, Sanofi Regeneron, Stallergenes Greer; fees for advisory boards or lecturing from AstraZeneca, Chiesi, GlaxoSmithKline, ALK, Allergy Therapeutics, Novartis, Sanofi Regeneron, Stallergenes Greer, HAL Allergy, Mundipharma Menarini, Malesci Guidotti, and Valeas. Janwillem W. H. Kocks: reports grants and personal fees from AstraZeneca, Boehringer Ingelheim, GlaxoSmithKline, and Novartis, personal fees from MSD and COVIS, grants from Chiesi, Mundipharma, and Teva outside the submitted work, and is a shareholder of the General Practitioners Research Institute. Asif H. Khan and Paul Rowe: employees of and stockholders in Sanofi. Siddhesh Kamat: employee of and stockholder in Regeneron Pharmaceuticals, Inc. At the time of development of this manuscript, Fen Ye was an employee of and stockholder in Sanofi and Yufang Lu was an employee of and stockholder in Regeneron Pharmaceuticals. Victoria Carter and Jaco Voorham (at the time of the study): employees of 
Observational and Pragmatic Research Institute Pte Ltd, which has conducted paid research in respiratory disease on behalf of the following organizations in the past 5 years: Aerocrine, AKL Research and Development Ltd, Almirall, AstraZeneca, Boehringer Ingelheim, Chiesi, GlaxoSmithKline, Meda, Mundipharma, Napp, Novartis, Orion, Takeda, Teva, and Zentiva (a Sanofi company). The authors report no other conflicts of interest in this work.

\section{References}

1. Robinson D, Humbert M, Buhl R, et al. Revisiting Type 2-high and Type 2-low airway inflammation in asthma: current knowledge and therapeutic implications. Clin Exp Allergy. 2017;47:161-175.

2. Global strategy for asthma management and prevention 2018; 2018. Available from: https://ginasthma.org/wp-content/uploads/2018/04/ wms-GINA-2018-report-V1.3-002.pdf. Accessed September 15, 2021.

3. Masoli M, Fabian D, Holt S, Beasley R. The global burden of asthma: executive summary of the GINA Dissemination Committee report. Allergy. 2004;59:469-478. doi:10.1111/j.1398-9995.20 04.00526.x

4. Gandhi NA, Bennett BL, Graham NM, Pirozzi G, Stahl N, Yancopoulos GD. Targeting key proximal drivers of type 2 inflammation in disease. Nat Rev Drug Discov. 2016;15:35-50. doi:10.1038/nrd4624

5. Corren J. Allergic rhinitis and asthma: how important is the link? $J$ Allergy Clin Immunol. 1997;99:S781-6. doi:10.1016/S00916749(97)70127-1

6. Seys SF, Scheers H, Van den Brande P, et al. Cluster analysis of sputum cytokine-high profiles reveals diversity in T(h)2-high asthma patients. Respir Res. 2017;18:39. doi:10.1186/s12931-017-0524-y

7. Peters MC, Mekonnen ZK, Yuan S, Bhakta NR, Woodruff PG, Fahy JV. Measures of gene expression in sputum cells can identify TH2-high and TH2-low subtypes of asthma. J Allergy Clin Immunol. 2014;133:388-394. doi:10.1016/j.jaci.2013.07.036

8. Diamont Z. Type 2 inflammation and the evolving profile of uncontrolled persistent asthma. 28th European Respiratory Society (ERS), International Congress in Paris: France; 2018. Available from: https:// emj.emg-health.com/wp-content/uploads/sites/2/2018/12/Type2-Inflammation-and-the-Evolving....pdf. Accessed September 15, 2021.

9. Global strategy for asthma management and prevention (update); 2019. Available from: https://ginasthma.org/wp-content/uploads/ 2019/06/GINA-2019-main-report-June-2019-wms.pdf. Accessed September 15, 2021.

10. Gershon AS, Wang C, Guan J, To T. Burden of comorbidity in individuals with asthma. Thorax. 2010;65:612-618. doi:10.1136/ thx.2009.131078

11. Chanoine S, Sanchez M, Pin I, et al. Multimorbidity medications and poor asthma prognosis. Eur Respir J. 2018;51(4):1702114. doi:10.1183/13993003.02114-2017

12. Jantunen J, Haahtela T, Salimäki J, et al. Multimorbidity in asthma, allergic conditions and COPD increase disease severity, drug use and costs: the Finnish Pharmacy Survey. Int Arch Allergy Immunol. 2019;179:273-280. doi:10.1159/000498861

13. Cazzoletti L, Marcon A, Corsico A, et al. Asthma severity according to Global Initiative for Asthma and its determinants: an international study. Int Arch Allergy Immunol. 2010;151:70-79. doi:10.1159/ 000232572
14. Chipps BE, Haselkorn T, Paknis B, et al. More than a decade follow-up in patients with severe or difficult-to-treat asthma: the Epidemiology and Natural History of Asthma: Outcomes and Treatment Regimens (TENOR) II. J Allergy Clin Immunol. 2018;141:1590-7 e9. doi:10.1016/j.jaci.2017.07.014

15. Matsusaka M, Kabata H, Fukunaga K, et al. Phenotype of asthma related with high serum periostin levels. Allergol Int. 2015;64:175-180. doi:10.1016/j.alit.2014.07.003

16. Schleich F, Brusselle G, Louis R, et al. Heterogeneity of phenotypes in severe asthmatics. The Belgian Severe Asthma Registry (BSAR). Respir Med. 2014;108:1723-1732. doi:10.1016/j. rmed.2014.10.007

17. Giavina-Bianchi P, Aun MV, Takejima P, Kalil J, Agondi RC. United airway disease: current perspectives. $J$ Asthma Allergy. 2016;9:93-100. doi:10.2147/JAA.S81541

18. Blöndal V, Malinovschi A, Sundbom F, et al. Multimorbidity in asthma, association with allergy, inflammatory markers and symptom burden, results from the Swedish GA(2) LEN study. Clin Exp Allergy. 2021;51:262-272. doi:10.1111/cea.13759

19. Oka A, Hirano T, Yamaji Y, et al. Determinants of incomplete asthma control in patients with allergic rhinitis and asthma. J Allergy Clin Immunol Pract. 2017;5:160-164. doi:10.1016/j.jaip.2016.08.002

20. OPCRD: an Introduction; 2017. Available from: http://opcrd.co.uk/ wp-content/uploads/2017/11/opcrd-brochure-final-21.11.17.pdf. Accessed September 15, 2021.

21. Chung KF, Wenzel SE, Brozek JL, et al. International ERS/ATS guidelines on definition, evaluation and treatment of severe asthma. Eur Respir J. 2014;43:343-373. doi:10.1183/09031936.00202013

22. Colice G, Chisholm A, Dima AL, et al. Performance of database-derived severe exacerbations and asthma control measures in asthma: responsiveness and predictive utility in a UK primary care database with linked questionnaire data. Pragmat Obs Res. 2018;9:29-42. doi:10.2147/POR.S151615

23. Noble M, Burden A, Stirling S. et al. Predicting asthma-related crisis events using routine electronic healthcare data. Br J Gen Pract. 2021. BJGP.2020.1042. doi:10.3399/BJGP.2020.1042

24. Tran TN, Kerkhof M, Carter V, Price DB. Persistence of eosinophilic asthma endotype and clinical outcomes: a real-world observational study. J Asthma Allergy. 2021;14:727-742. doi:10.2147/JAA.S306416

25. Ryan D, Heatley H, Heaney LG, et al. Potential severe asthma hidden in UK primary care. J Allergy Clin Immunol Pract. 2021;9:1612-23 e9. doi:10.1016/j.jaip.2020.11.053

26. Fahy JV. Type 2 inflammation in asthma - present in most, absent in many. Nat Rev Immunol. 2015;15:57-65. doi:10.1038/nri3786

27. Chen L, Grabowski KA, Xin JP, et al. IL-4 induces differentiation and expansion of Th2 cytokine-producing eosinophils. J Immunol. 2004;172:2059-2066. doi:10.4049/jimmunol.172.4.2059

28. Furuta GT, Aceves SS. The national biome initiative: an allergy perspective. $J$ Allergy Clin Immunol. 2017;139:1131-1134. doi:10.1016/j.jaci.2017.02.008

29. Peters $\mathrm{S}$. The impact of comorbid atopic disease on asthma: clinical expression and treatment. $J$ Asthma. 2007;44:149-161. doi:10.1080/ 02770900600925478

30. Price D, Zhang Q, Kocevar VS, Yin DD, Thomas M. Effect of a concomitant diagnosis of allergic rhinitis on asthma-related health care use by adults. Clin Exp Allergy. 2005;35:282-287. doi:10.1111/ j.1365-2222.2005.02182.x

31. Schatz M, Zeiger RS, Chen W, Yang SJ, Corrao MA, Quinn VP. The burden of rhinitis in a managed care organization. Ann Allergy Asthma Immunol. 2008;101:240-247. doi:10.1016/S1081-1206(10) 60488-7

32. Vandenplas O, Dramaix M, Joos G, et al. The impact of concomitant rhinitis on asthma-related quality of life and asthma control. Allergy. 2010;65:1290-1297. doi:10.1111/j.1398-9995.2010.02365.x 
33. Clatworthy J, Price D, Ryan D, Haughney J, Horne R. The value of self-report assessment of adherence, rhinitis and smoking in relation to asthma control. Prim Care Respir J. 2009;18:300-305. doi: $10.4104 /$ pcrj. 2009.00037

34. Steppuhn H, Langen U, Scheidt-Nave C, Keil T. Major comorbid conditions in asthma and association with asthma-related hospitalizations and emergency department admissions in adults: results from the German National Health Telephone Interview Survey (GEDA) 2010. BMC Pulm Med. 2013;13:46. doi:10.1186/1471-2466-13-46

35. Janson C, Lisspers K, Stallberg B, et al. Prevalence, characteristics and management of frequently exacerbating asthma patients: an observational study in Sweden (PACEHR). Eur Respir J. 2018;52: pii: 1701927. doi:10.1183/13993003.01927-2017

36. Illi S, von Mutius E, Lau S, et al. The natural course of atopic dermatitis from birth to age 7 years and the association with asthma. J Allergy Clin Immunol. 2004;113:925-931. doi:10.1016/j. jaci.2004.01.778

37. Skolnick HS, Conover-Walker MK, Koerner CB, Sampson HA, Burks W, Wood RA. The natural history of peanut allergy. $J$ Allergy Clin Immunol. 2001;107:367-374. doi:10.1067/ mai.2001.112129

38. Guerra S, Sherrill DL, Martinez FD, Barbee RA. Rhinitis as an independent risk factor for adult-onset asthma. $J$ Allergy Clin Immunol. 2002;109:419-425. doi:10.1067/mai.2002.121701

39. Shaaban R, Zureik M, Soussan D, et al. Rhinitis and onset of asthma: a longitudinal population-based study. Lancet. 2008;372:1049-1057. doi:10.1016/S0140-6736(08)61446-4

40. Boulet L-P, Boulay M-È. Asthma-related comorbidities. Expert Rev Respir Med. 2011;5:377-393. doi:10.1586/ers.11.34
41. Heck S, Al-Shobash S, Rapp D, et al. High probability of comorbidities in bronchial asthma in Germany. NPJ Prim Care Respir Med. 2017;27:28. doi:10.1038/s41533-017-0026-x

42. Castro M, Corren J, Pavord ID, et al. Dupilumab efficacy and safety in moderate-to-severe uncontrolled asthma. $N$ Engl J Med. 2018;378:2486-2496. doi:10.1056/NEJMoa1804092

43. Rabe KF, Nair P, Brusselle G, et al. Efficacy and safety of dupilumab in glucocorticoid-dependent severe asthma. $N$ Engl J Med. 2018;378:2475-2485. doi:10.1056/NEJMoa1804093

44. Marcus P, Arnold RJ, Ekins S, et al. A retrospective randomized study of asthma control in the US: results of the CHARIOT study. Curr Med Res Opin. 2008;24:3443-3452. doi:10.1185/ 03007990802557880

45. Boden SR, Burks AW. Anaphylaxis: a history with emphasis on food allergy. Immunol Rev. 2011;242:247-257. doi:10.1111/j.1600065X.2011.01028.X

46. Stevens WW, Schleimer RP, Kern RC. Chronic rhinosinusitis with nasal polyps. J Allergy Clin Immunol Pract. 2016;4:565-572. doi:10.1016/j.jaip.2016.04.012

47. Our Databases; 2020. Available from: https://opcrd.co.uk/ourdatabase/. Accessed September 15, 2021.

48. Wong MM, Keith PK. Presence of positive skin prick tests to inhalant allergens and markers of $\mathrm{T} 2$ inflammation in subjects with chronic spontaneous urticaria (CSU): a systematic literature review. Allergy Asthma Clin Immunol. 2020;16:72. doi:10.1186/s13223-020-00461-x

\section{Publish your work in this journal}

The Journal of Asthma and Allergy is an international, peer-reviewed open-access journal publishing original research, reports, editorials and commentaries on the following topics: Asthma; Pulmonary physiology; Asthma related clinical health; Clinical immunology and the immunological basis of disease; Pharmacological interventions and new therapies. The manuscript management system is completely online and includes a very quick and fair peer-review system, which is all easy to use. Visit http://www.dovepress.com/testimonials.php to read real quotes from published authors. 